\title{
Structural, Microhardness, Photoconductivity, and Dielectric Properties of Tris(thiourea) Cadmium Sulphate Single Crystals
}

\author{
A. P. Arthi, ${ }^{1}$ M. Sumithra Devi, ${ }^{2}$ and K. Thamizharasan ${ }^{3}$ \\ ${ }^{1}$ Department of Physics, Thangavelu Engineering College, Chennai 600 097, India \\ ${ }^{2}$ Department of Physics, Anand Institute of Technology, Chennai 603 103, India \\ ${ }^{3}$ Department of Physics, Sir Theagaraya College, Chennai 600 021, India
}

Correspondence should be addressed to K. Thamizharasan; tamilsurya@yahoo.in

Received 24 August 2014; Revised 4 November 2014; Accepted 4 November 2014; Published 20 November 2014

Academic Editor: Shi J. Xu

Copyright (C) 2014 A. P. Arthi et al. This is an open access article distributed under the Creative Commons Attribution License, which permits unrestricted use, distribution, and reproduction in any medium, provided the original work is properly cited.

\begin{abstract}
Semiorganic nonlinear optical tris(thiourea) cadmium sulphate (TTCS) single crystals were grown by slow evaporation method. The crystal system, cell parameter of the grown crystal, was identified by powder X-ray diffraction study. The self-focusing $Z$-scan technique has been employed to observe the third-order nonlinear optical property of the grown crystal. The mechanical property of the grown crystal was examined by using Vicker's microhardness test. Chemical etching studies were made on the TTCS crystal using water as an etchant. The dark current and photocurrent properties of the crystal were estimated by using photoconductivity study. The dielectric constant of grown crystal was studied in different temperature by varying applied frequencies.
\end{abstract}

\section{Introduction}

The semiorganic nonlinear optical (NLO) materials have a significant impact on laser technology, optical communication, and are applied in optical storage technologies in recent years $[1,2]$. The organic materials have large NLO coefficient when compared to inorganic materials, but their usage is impeded due to their poor mechanical strength, low thermal stability, and low laser damage threshold [3]. This organic molecular salt exhibits interesting NLO properties because of its strong Coulomb interactions between charged molecules [4]. The inorganic materials have excellent mechanical and thermal properties but possess relatively modest optical nonlinearities, due to lack of extended $\pi$-electron delocalization [5]. Both the high nonlinear optical efficiency and stable materials are the interest of the future technological advancements. The semiorganic material offers great materials for second- and third-order nonlinear optical applications. The solution growth technique is the efficient way to produce good quality semiorganic NLO crystals [6]. One such semiorganic material is the metal complex of thiourea. The thiourea molecule is an interesting inorganic matrix modifier because of its large dipole moment. It has good ability to form an extensive network of hydrogen bond and has the coordination capacity to form different phases of metal-thiourea complexes $[7,8]$. In our study, thiourea a typical polar molecule was selected to combine with cadmium sulphate and its results are summarized.

\section{Experimental Work}

The semiorganic nonlinear optical compound tris(thiourea) cadmium sulphate (TTCS) was synthesized by direct chemical reaction. The calculated amount of AR grade thiourea ( $3 \mathrm{~mol} \%)$ and cadmium sulphate $(1 \mathrm{~mol} \%)$ was dissolved in deionized water. The mixtures of the reactants were stirred well for about $6 \mathrm{~h}$ to avoid coprecipitation of multiple phases. The synthesized solution produced TTCS salt and its purity was improved by repeated recrystallization processes in aqueous solution. This purified salt was then used to prepare saturated TTCS solution that was further stirred well and filtered with high quality filter paper to avoid any impurity. The saturated growth solution was poured into a container and isolated for inciting slow evaporation of the solvent. After 3 weeks, the supersaturated mother solution yields the spontaneously nucleated TTCS crystals and the well-grown 


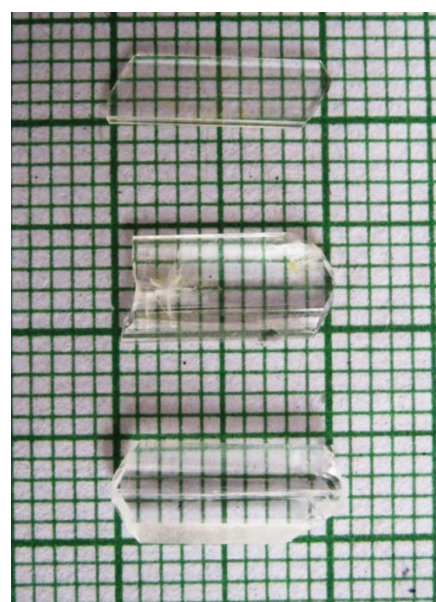

FIGURE 1: As-grown TTCS crystal by slow evaporation method.

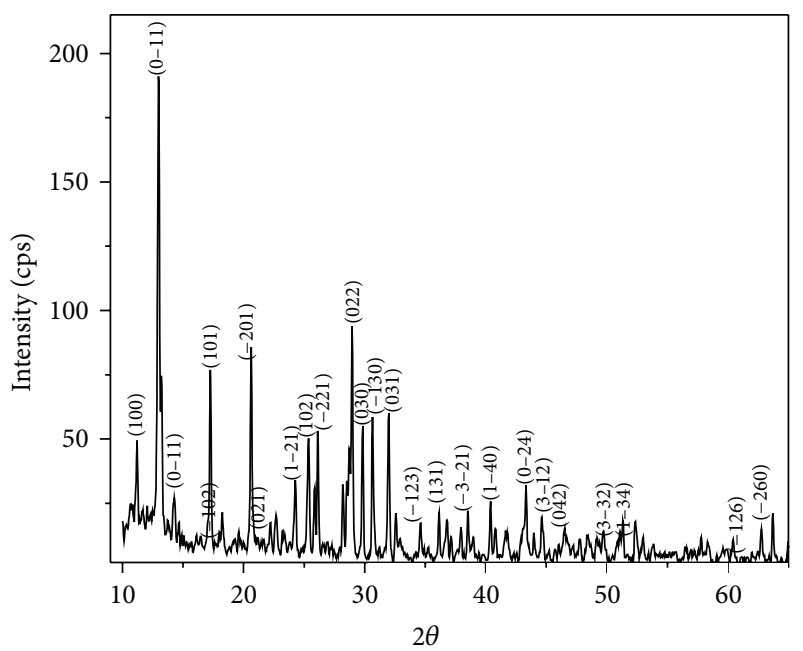

Figure 2: Powder X-ray diffraction pattern of TTCS crystal.

TTCS crystals were harvested in a period of 4-5 weeks. The optically transparent TTCS crystals, whose dimension is up to $10 \times 5 \times 4 \mathrm{~mm}^{3}$, were obtained as shown in Figure 1. The good quality crystals were used for further characterizations.

\section{Results and Discussion}

3.1. X-Ray Diffraction Study. The powder X-ray diffraction pattern of TTCS crystal was recorded by SIEFERT X-ray diffractometer using $\operatorname{Cuk}_{\alpha}\left(\mathrm{k}_{\alpha}=1.54 \AA\right)$ radiation. The recorded powder X-ray diffraction pattern of TTCS crystal is shown in Figure 2. The disclosure of well-defined Bragg's peaks at specific $2 \theta$ angle shows the high crystallinity of TTCS crystals. The cell parameters of the as-grown TTCS single crystals were evaluated. The values belonged to triclinic system of centrosymmetric $\overline{P_{1}}$ space group, with cell dimensions of $a=8.71 \AA, b=9.04 \AA, c=9.73 \AA, \alpha=91.76^{\circ}, \beta=110.58^{\circ}$, and $\gamma=95.55^{\circ}$ and volume $(V)=713 \AA^{3}$. The observed cell values agreed with reported values $[9,10]$.
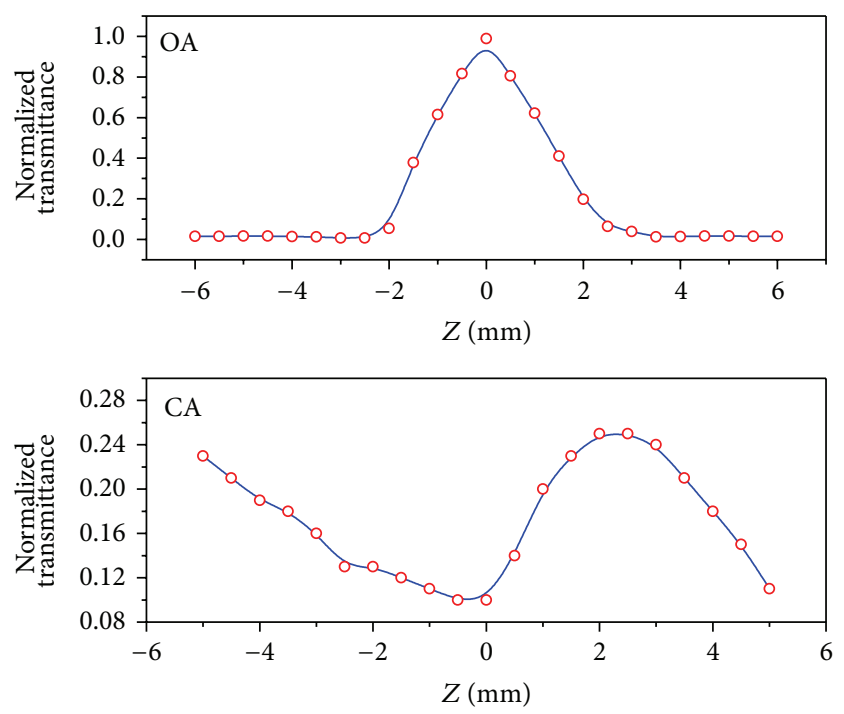

FIgURE 3: Open aperture (OA) and closed aperture (CA) $Z$-scan curves of TTCS crystal.

3.2. Z-Scan Measurement. Third-order nonlinear optical property of TTCS crystal was studied by $Z$-scan technique. The magnitude and sign of the nonlinear refractive index $\left(n_{2}\right)$ and nonlinear absorption coefficient $(\beta)$ of the crystals were calculated from the $Z$-scan data [11-13]. The open aperture $(\mathrm{OA})$ and closed aperture (CA) $Z$-scan methods are used for the measurement of nonlinear absorption coefficient and nonlinear optical refraction for optical materials. In this experiment, Gaussian laser beam was used for molecular excitation and its propagation direction has been taken as the $Z$-axis of the optically polished crystal. The beam was focused using a convex lens and the focal point has been taken as $Z=0$. The monochromatic continuous wave $(\mathrm{cw})$ laser light $632.8 \mathrm{~nm}$ with power of $20 \mathrm{~mW}$ beam from $\mathrm{He}-\mathrm{Ne}$ laser was used. The optically polished $1 \mathrm{~mm}$ thick crystal sample was fixed in the travel range of $12 \mathrm{~mm}$. The input energy and the energy transmitted by the sample were measured using a power meter. The normalized transmittance for the positioned crystal sample was measured at different positions and was used to calculate third-order nonlinear optical property of the crystal. The peak followed by a valley of normalized transmittance is the signature for nonlinearity of the material. The $Z$-scan curves in closed and open aperture modes are illustrated in Figure 3. It reveals that nonlinear refractive index $n_{2}=1.83 \times 10^{-11} \mathrm{~m}^{2} \mathrm{~W}$ and nonlinear absorption coefficient $\beta=1.39 \times 10^{-5} \mathrm{~cm} / \mathrm{W}$. The imaginary and real parts of the third-order susceptibility values were determined as $\operatorname{Im} \chi^{3}=9.67 \times 10^{-12}$ esu and $\operatorname{Re} \chi^{3}=1.5974 \times 10^{-11}$ esu, respectively.

3.3. Vicker's Microhardness Test. The hardness property of the crystals played a key role in device fabrication. The transparent crystals, free from cracks, were selected for microhardness measurement. Microhardness test for the grown crystals was carried out using a Leitz Metallux-II microscope with a calibrated ocular at the magnification of "X500." Vicker's 


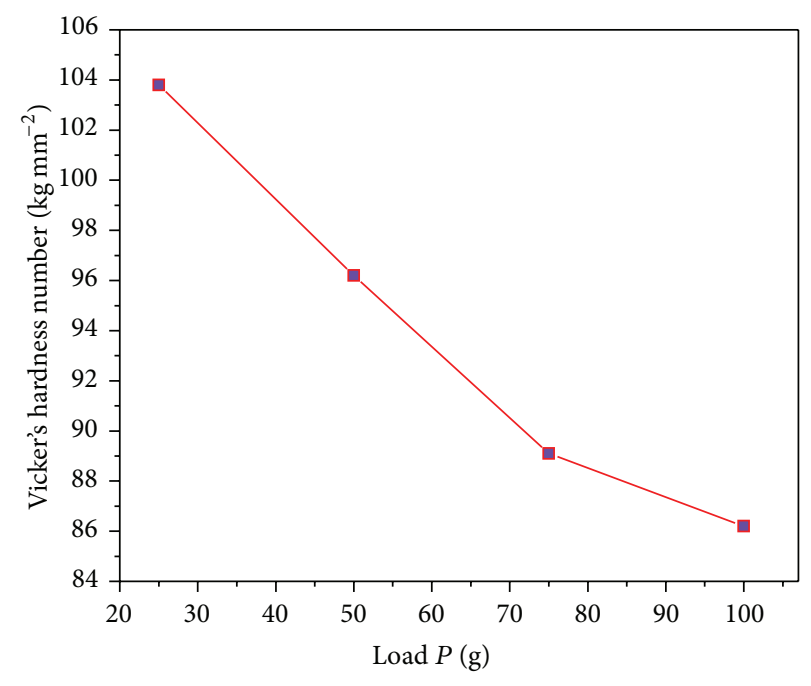

(a)

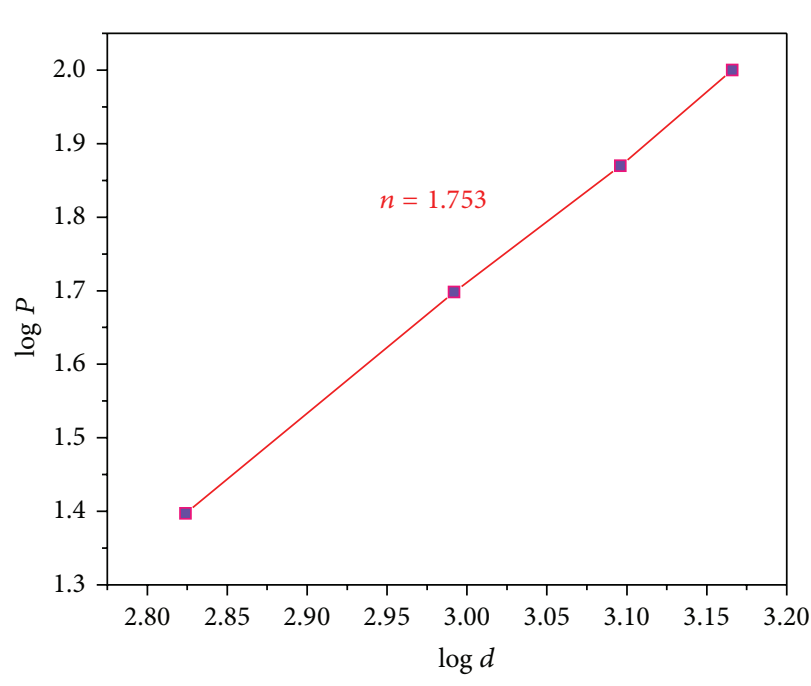

(b)

FIgURE 4: (a) Variation of Vicker's microhardness number $\left(H_{V}\right)$ with applied load $(P)$ for TTCS crystal. (b) Variation between log $P$ and $\log d$ of TTCS crystal.

microhardness study had been used to analyze the hardness property of the grown TTCS crystals using a Leitz microhardness tester fitted with a diamond pyramidal indenter. A well-polished TTCS crystal was used for the study and it was placed on the platform of Vicker's microhardness. The loads $(P)$ of different magnitudes were applied over a fixed interval of time with an indentation time of $8 \mathrm{~s}$ for all the loads. The hardness number was calculated using the relation

$$
H_{V}=1.8544 \frac{P}{d^{2}} \mathrm{Kg} / \mathrm{mm}^{2},
$$

where " $H_{V}$ " is the Vicker's microhardness number, " $P$ " is the applied load in "Kg," and " $d$ " is the diagonal length of the indentation impression. The plot between hardness number $\left(H_{V}\right)$ and applied load $(P)$ is shown in Figure 4(a) and it shows that hardness number decreases with increase in applied load. The Meyer's index $(n)$ is used to determine if the material belonged to soft category or hard category. According to Onitsch [14], the value $(n)$ ranges between 1 and 1.6 for hard materials and is greater than 1.6 for soft materials. The Meyer's index number was calculated using Meyer's law as follows:

$$
\begin{gathered}
P=K d^{n}, \\
\log P=\log k+n \log d,
\end{gathered}
$$

where " $k$ " is the constant for a material and " $n$ " is the Meyer's index. In order to find the value of " $n$," a graph was plotted between applied loads " $\log P$ " and " $\log d$," which resulted in the formation of a straight line. The slope value of the plot in Figure 4 (b) was identified as $n=1.753$, thus confirming that the TTCS crystal belonged to soft category.

3.4. Chemical Etching. The NLO property of the crystal purely depends on the perfection of the grown crystal. Microstructure analysis was carried out on the grown crystal by

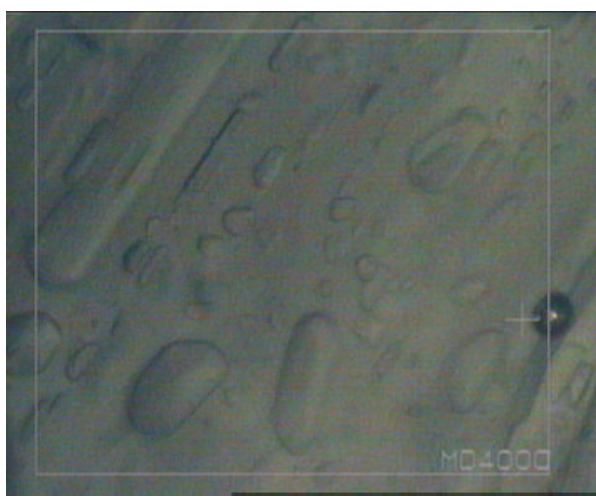

FIGURE 5: Etch pit pattern of TTCS crystal with water in $10 \mathrm{~s}$.

using optical microscope in the reflective mode. The wellpolished crystal was used for surface treatment using water as an etchant in 10 seconds. The atoms at the grain boundaries are chemically more active, consequently dissolve more readily, than those within the grain forming small grooves. The grooves become discernible when viewed under microscope because of reflected light with different angle. The recorded etch pit pattern of TTCS crystal is shown in Figure 5 in the scale of $100 \mu \mathrm{m}$. From the etching analysis revealed that the rod like etchpit pattern observed in TTCS crystal.

3.5. Photoconductivity Studies. Photoconductivity is the incremental change in the electrical conductivity of a substance upon illumination which is generated by the absorption of photons [15]. The relevant photoexcitation of free carriers and photoconductivity are expected when the crystal is illuminated with visible or near-infrared wavelengths. The polished, defect-free TTCS crystal sample which was rectangular in size was used for the study. Photoconductivity property of the grown TTCS crystal was measured in the 


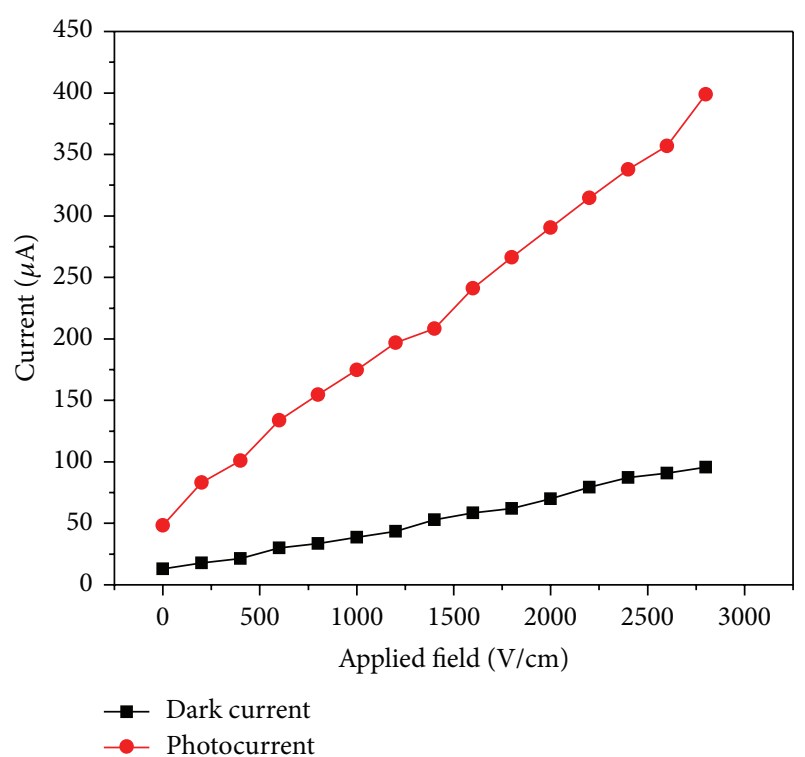

FIGURE 6: Photoconductivity measurement of TTCS crystal.

range of $0-2800 \mathrm{~V} / \mathrm{cm}$ using a Keithley picoammeter. The electrical contacts were made with the sample by silver paint and it was connected by copper wire that was used as an electrode. The light from halogen lamp of $100 \mathrm{~W}$ was focused on the sample using convex lens. In addition, the applied voltage was increased by $0-2800 \mathrm{~V}$ and the corresponding photocurrent was measured with respect to the applied voltage. The variations of photocurrent $\left(I_{P}\right)$ and dark current $\left(I_{d}\right)$ with applied field were shown in Figure 6. It was observed that both dark current and photocurrent of the crystals increase linearly with the applied electric field but if the dark current is less than the photocurrent, photoconductivity of the TTCS crystal is positive

3.6. Dielectric Constant. The dielectric constant of crystal is due to the contribution of electronic, ionic, orientation, and space charge polarization, which are predominant in the lower frequency region [16]. Dielectric measurements of the crystals were carried out using a HIOKI model 3532-50 LCR HITESTER. The dielectric measurements were carried out from $40 \mathrm{~K}$ to $150 \mathrm{~K}$ at the frequencies of $1 \mathrm{KHz}-1 \mathrm{MHz}$ and were shown in Figure 7. It was noticed that variation of dielectric constant as a function of frequency suggests that the dielectric constant is relatively higher in the low frequency region. The dielectric constant of the crystal decreases when frequency increases. The characteristic of lower dielectric constant with fewer defects crystal is utilized for various NLO applications.

\section{Conclusion}

Semiorganic thiourea metal complex, tris(thiourea) cadmium sulphate single crystals with dimension up to $10 \times 5 \times$ $4 \mathrm{~mm}^{3}$, was grown by slow evaporation technique. Powder Xray diffraction study confirmed the cell dimension values and structure of the lattice. Third-order nonlinear refractive index

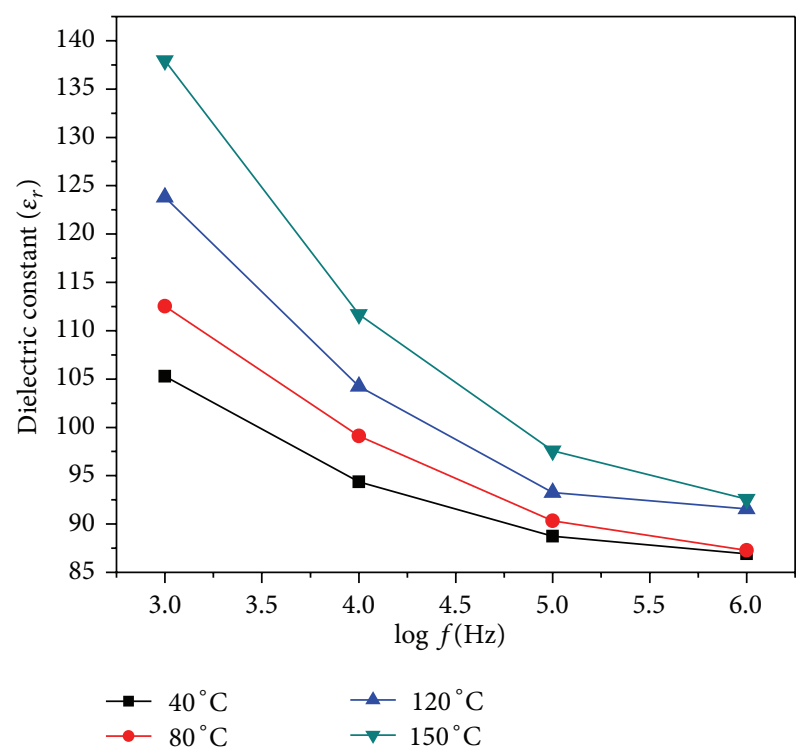

Figure 7: Dielectric constant versus $\log f$ of TTCS crystal.

$\left(n_{2}=1.83 \times 10^{-11} \mathrm{~m}^{2} \mathrm{~W}\right)$, nonlinear absorption coefficient $\left(\beta=1.39 \times 10^{-5} \mathrm{~cm} / \mathrm{W}\right)$, and third-order nonlinear optical susceptibility $\left(\chi^{3}=1.189 \times 10^{-8} \mathrm{esu}\right)$ were estimated by $Z$ scan technique. The hardness property of the crystal was studied and its Meyer's index value $n=1.753$ suggested that TTCS is a soft material. The microstructure of the growth pattern was analyzed using etch pit study. The high value of photocurrent was observed than the dark current for different applied fields. The dielectric constant of the crystal was found to be high in the low frequency region and decreased with increase in applied frequency. All the results explained its usefulness to the optical applications.

\section{Conflict of Interests}

The authors declare that there is no conflict of interests regarding the publication of this paper.

\section{References}

[1] H. O. Marey, L. F. Warns, M. S. Webb et al., "Second-harmonic generation in zinc tris(thiourea) sulfate," Applied Optics, vol. 31, no. 24, pp. 5051-5060, 1992.

[2] X. Q. Wang, D. Xu, D. R. Yuan et al., "Synthesis, structure and properties of a new nonlinear optical material: zinc cadmium tetrathiocyanate," Materials Research Bulletin, vol. 34, no. 12, pp. 2003-2011, 1999.

[3] M. Jiang and Q. Fang, "Organic and semiorganic nonlinear optical materials," Advanced Materials, vol. 11, pp. 1147-1151, 1999.

[4] A. K. Dharmadhikari, B. Roy, S. Roy, J. A. Dharmadhikari, A. Mishra, and G. R. Kumar, "Higher-order optical nonlinearities in $4^{\prime}$-dimethylamino- $N$-methyl-4-stilbazolium tosylate," Optics Communications, vol. 235, no. 1-3, pp. 195-200, 2004.

[5] J. Ramajothi, S. Dhanuskodi, and K. Nagarajan, "Crystal growth, thermal, optical and microhardness studies of tris 
(thiourea) zinc sulphate-a semiorganic NLO material," Crystal Research and Technology, vol. 39, no. 5, pp. 414-420, 2004.

[6] N. Zaitseva, L. Carman, A. Glenn et al., "Application of solution techniques for rapid growth of organic crystals," Journal of Crystal Growth, vol. 314, no. 1, pp. 163-170, 2011.

[7] S. G. Bhat and S. M. Dharmaprakash, "A new metal-organic crystal: bismuth thiourea chloride," Materials Research Bulletin, vol. 33, no. 6, pp. 833-840, 1998.

[8] G. A. Bowmaker, J. V. Hanna, C. Pakawatchai, B. W. Skelton, Y. Thanyasirikul, and A. H. White, "Crystal structures and vibrational spectroscopy of copper(l) thiourea complexes," Inorganic Chemistry, vol. 48, no. 1, pp. 350-368, 2009.

[9] L. Cavaica, A. C. Villa, A. Mangia, and C. Palmeiri, "The crystal structure of tris(thiourea)cadmium sulphate," Inorganica Chimica Acta, vol. 4, pp. 463-470, 1970.

[10] E. Corao and S. Baggio, "The crystal structure of a five-coordinated cadmium(II) complex: Tristhiourea-cadmium sulphate," Inorganica Chimica Acta, vol. 3, pp. 617-622, 1969.

[11] R. DeSalvo, M. Sheik-Bahae, A. A. Said, D. J. Hagan, and E. W. Van Stryland, "Z-scan measurements of the anisotropy of nonlinear refraction and absorption in crystals," Optics Letters, vol. 18, no. 3, pp. 194-196, 1993.

[12] M. Krishna Kumar, S. Sudhahar, P. Pandi, G. Bhagavannarayana, and R. Mohan Kumar, "Studies of the structural and third-order nonlinear optical properties of solution grown 4hydroxy-3-methoxy-4' - $\mathrm{N}^{\prime}$-methylstilbazolium tosylate monohydrate crystals," Optical Materials, vol. 36, no. 5, pp. 988-995, 2014.

[13] M. K. Kumar, S. Sudhahar, A. Silambarasan, B. M. Sornamurthy, and R. M. Kumar, "Crystal growth, structural, linear and nonlinear optical studies of 4 -methyl- $4^{\prime}-\mathrm{N}^{\prime}$-methylstilbazolium tosylate single crystals," Optik, vol. 125, no. 2, pp. 751-755, 2014.

[14] E. M. Onitsch, "The present status of testing the hardness of materials," Microscope, vol. 95, pp. 12-14, 1950.

[15] S. Follonier, M. Fierz, I. Biaggio, U. Meier, C. Bosshard, and P. Günter, "Structural, optical, and electrical properties of the organic molecular crystal 4-N,N-dimethylamino- $4^{\prime}-\mathrm{N}^{\prime}$-methyl stilbazolium tosylate," Journal of the Optical Society of America B: Optical Physics, vol. 19, no. 9, pp. 1990-1998, 2002.

[16] C. Balarew and R. Dehlew, "Application of the hard and soft acids and bases concept to explain ligand coordination in double salt structures," Journal of Solid State Chemistry, vol. 55, no. 1, pp. 1-6, 1984. 

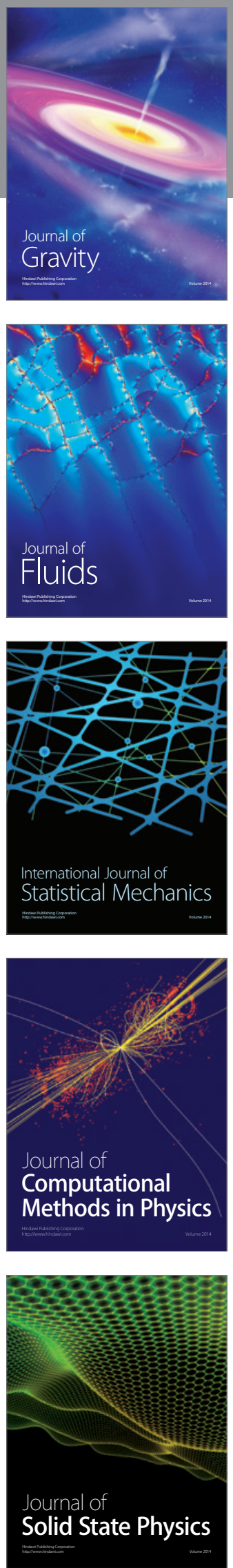

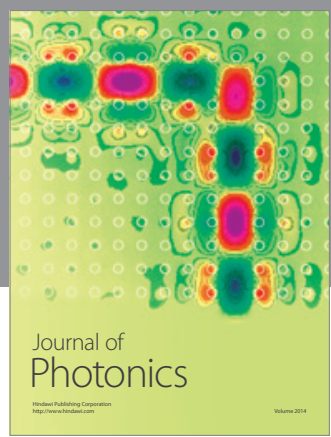

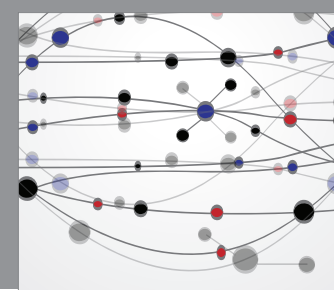

The Scientific World Journal

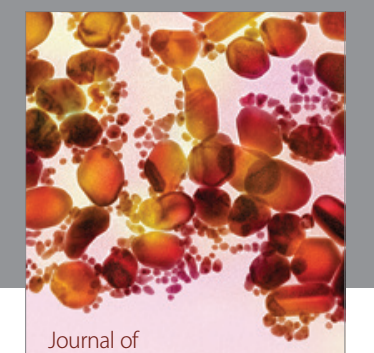

Soft Matter
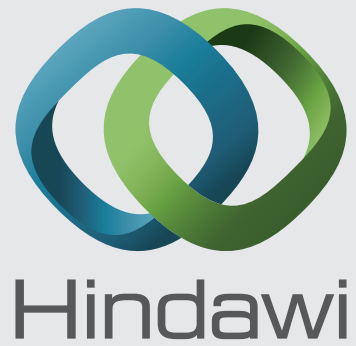

Submit your manuscripts at

http://www.hindawi.com
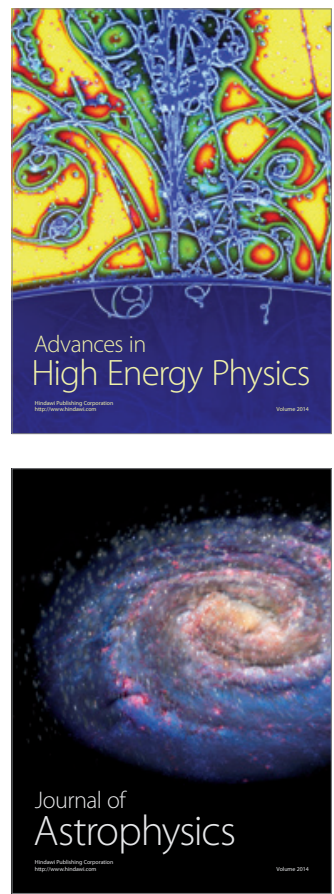
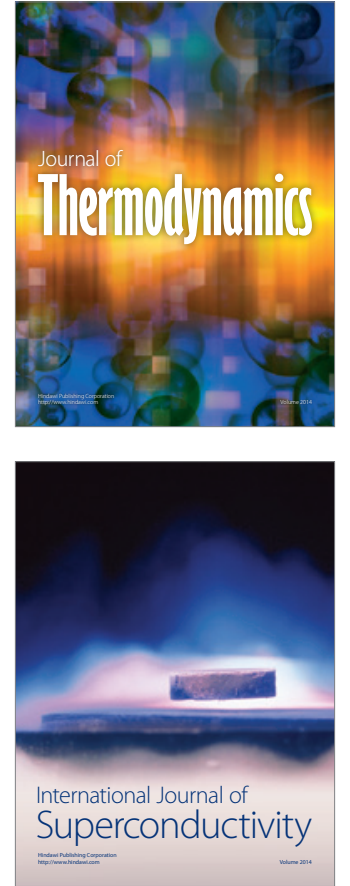
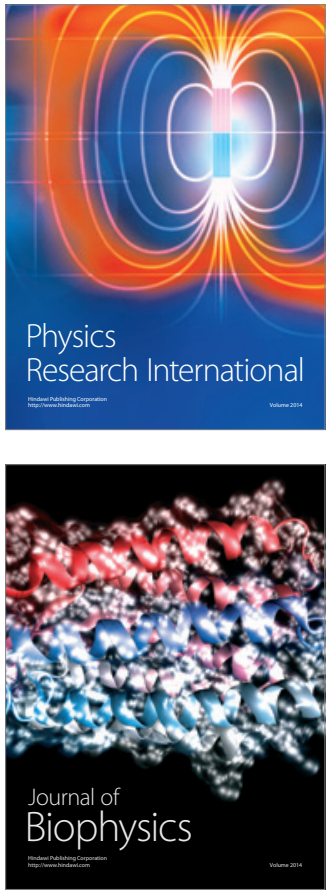
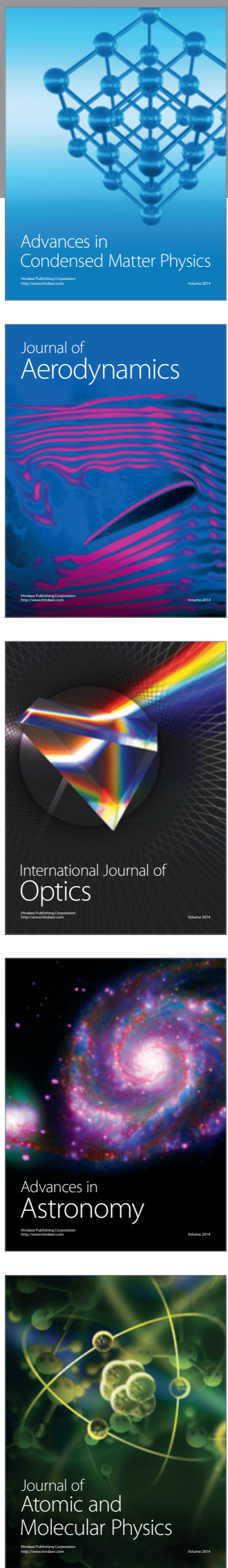